\title{
Improving the uptake and comprehensiveness of bedside cognitive testing amongst liaison psychiatrists over an eight-month period.
}

\author{
Ruaidhri McCormack
}

St Thomas's Hospital, London, UK.

\begin{abstract}
The aim of this quality improvement (QI) project was to improve the uptake and comprehensiveness of bedside cognitive testing amongst liaison psychiatrists over an eight month period.

The baseline measurement involved an audit of the practice of the neuropsychiatry liaison team over six months at the 840 bed St Thomas's Hospital in London, UK. Of 35 referrals, 21 patients were able, and suitable, for cognitive testing based on the referral data and clinical interview. Fourteen (66.6\%) of these patients had an Addenbrooke's Cognitive Examination (ACE), while 5 (23.8\%) had frontal testing. The frontal tests performed were variable and inconsistent.
\end{abstract}

Two Plan-Do-Study-Act (PDSA) cycles were then conducted. Clear guidance was issued to the team on the use of the ACE or Montreal Cognitive Assessment (MoCA) in suitable patients, and how these can be supplemented by frontal testing. Given the inconsistency in the types and combination of frontal tests being conducted at baseline, a frontal lobe test sheet containing established tests was developed and implemented. In PDSA cycle 1, $100 \%(n=10)$ of able and suitable referrals had an ACE or MoCA while $100 \%$ had frontal testing $(80 \%$ of these using the dedicated test sheet). In PDSA cycle 2, improvements were broadly maintained with $85.7 \%(n=6)$ of referrals having an ACE/MoCA and $85.7 \%$ having frontal testing ( $83.3 \%$ of these using the dedicated frontal test sheet). In conclusion, our team improved the uptake and comprehensiveness of bedside cognitive testing by changing existing practice with clear protocols regarding the use of the ACE/MoCA and the implementation of a frontal test sheet.

\section{Problem}

The neuropsychiatry liaison team believed we could improve prevailing practice with regards to bedside cognitive testing. It was our opinion that we should be able to offer comprehensive bedside cognitive assessment given the specialist nature of our service. This was notwithstanding any availability of neuropsychology review for select cases. With this in mind, we audited the team's practice to see if our informal opinions about the possibility of improvement were reflected in real practice. This was with the intention of directly improving the quality of patient assessment.

One further problem is that many of the most common bedside cognitive tools have limitations. The tools available do not necessarily cover all areas of memory and cognition. This extends even to the Addenbrooke's Cognitive Examination (ACE), which is well regarded. We needed to look at this, and make judgements about whether or not tests could be combined to facilitate comprehensive assessment.

\section{Background}

St Thomas's Hospital is an 840 bed general hospital operated by Guys and St Thomas' NHS Foundation Trust in the London borough of Lambeth. The hospital has a large liaison psychiatry department designed to service the needs of medical and surgical patients in the emergency department and on the wards. This includes a general adult liaison psychiatry team plus liaison subspecialties including older adults, perinatal, child and adolescent, and neuropsychiatry liaison psychiatry.

At the time of this project, the neuropsychiatry liaison team comprised two neuropsychiatry consultants, two higher trainees (one full-time, one half-time), and one full-time core trainee. Junior trainees also have some responsibility to the broader general adult liaison service. The neuropsychiatry team sees cases where there are psychiatric symptoms in combination with a high suspicion of, or actual confirmed, underlying neurological disease. In this context, the team often sees cases where deficits in cognition and memory are key aspects of the presentation. These deficits may be the result of brain injury, or the product of a pseudodementia in the context of underlying psychiatric illness. Brain injury has a myriad of potential aetiologies including the dementias, traumatic injury, stroke, alcohol, or other neurodegenerative, infectious, and inflammatory conditions.

It is important to detect, measure, and map any prevailing cognitive deficits so as to help inform impressions regarding diagnosis, daily functioning, prognosis, and potential for engagement in (and the design of) rehabilitation programmes. Serial cognitive measures can also be used to inform judgements regarding response to treatment in cases where the aetiology is reversible. Patients and families want to know how they will be directly affected, and what to expect. 
BMJ Quality Improvement Reports

In the liaison setting, patients have been admitted to hospital owing to active acute medical or surgical illness and they are often quite morbid. Considering this, it is not always feasible to conduct formal neuropsychological testing due to the time and energy required by the patient. In this setting, the team should be able to adapt and conduct a structured yet more tolerable form of bedside cognitive testing.

NICE guidance (CG42) for dementia suggests that each patient should have an examination of attention and concentration, orientation, short and long-term memory, praxis, language, and executive function.[1] NICE recommend the use of a 'standardised instrument' like the 6-item Cognitive Impairment Test (6-CIT), the General Practitioner Assessment of Cognition (GPCOG), or the 7-Minute Screen.[1] These screening tools are limited in that they are very short and do not provide a significant level of detail on the integrity or balance of individual cognitive functions (e.g. visuospatial skill, language skill). This guidance is also only intended for patients with dementia. In her 2007 review paper, Cullen provides a very good overview of the tools currently available for bedside testing and their limitations.[2]

The Addenbrooke's cognitive examination (ACE-III) is one of the most comprehensive standardised and widely validated bedside cognitive assessment tools available.[3] It takes 15-20 minutes to administer (although can take longer in those with significant cognitive deficits). It gives an overall score out of 100 , with good sensitivity and specificity for dementia at cut-off scores of 82-88. It also gives a breakdown of scores in select domains of cognition attention/orientation, language, visuospatial skill, fluency, and memory. The ACE has however been criticised for not including comprehensive tests of frontal or executive function, nor does it have much if any testing of visual memory, the apraxias or the agnosias. It is also quite a language-based test. On balance, however, the ACE is considered the test of choice in our service for bedside cognitive testing. The Montreal Cognitive Assessment (MoCA) is a shorter screening tool scored out of 30 , which can be used in patients who cannot tolerate an ACE, and avoids the copyright concerns associated with the Mini-Mental State Examination (MMSE).[4-6]

The primary aim of this QI project was to improve the uptake and comprehensiveness of bedside cognitive testing amongst liaison psychiatrists. The aims in detail were:

1. To assess prevailing practice with regards to bedside cognitive testing.

2. To identify the limitations of the current cognitive assessment tool(s).

3. To implement $+/$ - develop a cognitive assessment tool that might address said limitations for use by clinicians at the bedside.

4. To distribute the cognitive assessment tool for use amongst clinicians.

5. To re-assess the uptake and comprehensiveness of bedside cognitive testing in the same liaison neuropsychiatry service over two time-periods thereby intending to demonstrate improvements (or if not, identify the reasons for lack of improvement).

\section{Baseline measurement}

The baseline measurement looked at all patients referred to the neuropsychiatry inpatient liaison service at St Thomas's Hospital over a six month period (10th November 2014 through 10th May 2015).

The data extracted from the trust's electronic records included:

1. Age and Gender.

2. Retrospective clinical judgement on whether or not cognitive testing would have been useful - were patients suitable for testing?

3. Any insurmountable barriers to cognitive testing - were patients able for testing?

4. The type of bedside cognitive testing that actually occurred (Addenbrookes yes/no, subcategories of Addenbrooke's documented, frontal tests yes/no [and specify which tests], other tests yes/no [and specify which tests]).

The data were extracted and then analysed.

Over the 6 month period studied, 39 referrals (37 patients with 2 patients being referred twice) were accepted for review by the neuropsychiatry liaison team. Of the 37 patients, 26 (70.3\%) were male and $11(29.7 \%)$ female, with mean age across the whole group being 53.0 years.

Of the 39 referral episodes, 35 (89.7\%) referrals were retrospectively considered to have been suitable for testing - that is, that cognitive testing would have helped inform the diagnostic formulation or management plan. Fourteen $(40 \%)$ of the 35 suitable referrals had insurmountable barriers to testing, with many having multiple barriers. These, in order of frequency, included:

1. Active affective/psychotic axis-1 psychiatric diagnosis affecting score validity and/or performance of tests $(n=10$, $28.6 \%$ of eligible referrals)

2. Patient too unwell $(n=7,20.0 \%)$

3. Patient refused $(n=2,5.71 \%)$

Other barriers included the patient having a primary illiteracy prohibiting many tests $(n=1,2.86 \%)$, the patient being unable to communicate in any reasonable way $(n=1,2.86 \%)$, the patient having a language barrier in combination with other barriers listed $(n=1,2.86 \%)$.

Of the 21 remaining referrals able and suitable for testing, 14 (66.6\%) had an ACE +/- other tests. Thirteen of these 14 (92.9\%) had adequate documentation of the subcategory performance in the ACE.

Beyond the ACE, five (23.8\%) of the 21 referrals able and suitable for testing also had a frontal assessment performed. The tests included in the frontal assessment were variable and inconsistent, usually consisting of some limited combination of verbal fluency, 
similarities, cognitive estimates, proverb interpretation, and the Luria motor test. Tests never included the go-no-go test. One of these patients had a frontal assessment battery (FAB) done. Two of the 21 referrals $(9.52 \%)$ had documented tests for apraxia. One of the 21 referrals $(4.76 \%)$ had a more detailed parietal lobe cognitive examination with left-right orientation, finger recognition, astereognosis, and dysgraphaesthesia tests performed.

\section{Design}

From the baseline measurement data, we established that the majority of patients referred to the neuropsychiatry liaison team are able and suitable for bedside cognitive testing. There are however often barriers to performing cognitive testing in the liaison setting.

It was clear that there was considerable room for improvement in the uptake of bedside cognitive testing. The ACE was not done in a third $(n=7)$ of able and suitable referrals. Alternative tests were not being employed (e.g. the MoCA) when the ACE was too challenging for the patient. The testing done was not sufficiently comprehensive, with frontal testing only occurring in $23.8 \%(n=5)$. The use of tests of apraxia and parietal lobe function was even lower. When frontal or parietal tests were performed, the individual tests varied and were inconsistent.

It was therefore recommended that an ACE be done on all able and suitable referrals. If an ACE was too challenging, a MoCA should be used. In addition, a frontal/executive test sheet was drawn up to include - verbal fluency, proverbs, cognitive estimates, similarities, the Luria motor test, the go-no-go testing, working memory digitspan tests (forward and reverse), and graphical sequencing. This test sheet did not contain new tests - rather, it contained established tests in an organised fashion to facilitate ease of testing.

\section{Strategy}

Following baseline measurement, two PDSA cycles were undertaken.

PDSA Cycle 1: A frontal test sheet was designed to include - verbal fluency, proverbs, cognitive estimates, similarities, the Luria motor test, the go-no-go test, working memory digit-span tests (forward and reverse), and graphical sequencing. Advice was given to implement this along with an ACE or a MoCA (if ACE not feasible) in able and suitable referrals.

PDSA Cycle 2: Given the successful results in PDSA cycle 1 (see below), the same intervention was recommended. Discussion took place regarding a further parietal lobe test sheet but it was considered by the team that this would be too time-consuming and that more detailed parietal lobe testing should take place on a caseby-case basis in accordance with clinical judgement/indication.

Dissemination of Results: The results of both PDSA cycle 1 and PDSA cycle 2 were disseminated locally by email and face-to-face team meetings. Now that both cycles are completed, the methods and results are also in the process of being disseminated at national and international levels by way of an abstract and poster publication at the Royal College of Psychiatrist's International Congress (pending acceptance at the time of writing this paper) and a publication in the journal BMJ Quality Improvement Reports.

\section{Post-measurement}

PDSA cycle 1: Following the measurement and analysis of baseline data, the intervention was to instruct team members to (a) conduct an ACE or MoCA on all able and suitable referrals (b) use the newly designed frontal test sheet on all patients. All results were to be documented on the trust electronic records system, and any barriers to testing similarly documented. The measures were similar to those taken in the collection of baseline data - age, gender, retrospective judgement on usefulness of testing, identification of any insurmountable barriers to cognitive testing, whether patient had an ACE/MoCA documented (with subcategories of ACE documented if applicable), occurrence of frontal testing (and uptake of the newly designed frontal test sheet). This was done over the course of a month (June 2015). We predicted an improvement in the uptake of bedside cognitive testing.

The results were as follows. There were 19 referrals in total, with mean age 56.2 years, 10 (52.6\%) being male. Sixteen (84.2\%) referrals were suitable for testing although six (37.5\%) of these had insurmountable barriers to testing including severity of medical or psychiatric illness, patient refusal, and severe aphasia. Of the 10 able and suitable referrals, all (100\%) had either an ACE or MoCA, an ACE being done in $80 \%(n=8)$ of these and a MoCA in $20 \%$ $(n=2)$. Where applicable, the ACE cognitive subcategories were documented in all (100\%) cases. All $10(100 \%)$ had frontal testing, the frontal test sheet being used in $80 \%(n=8)$ of these cases.

These results were disseminated to the team by email and face-toface meeting. Following discussion, it was decided that adding additional tests to the current battery of routine tests (e.g. parietal lobe tests) would be too time-consuming and take away from other activities in a busy liaison service. It was therefore decided that further tests would not be conducted routinely, rather only on the basis of individual clinical need.

From this PDSA cycle therefore, the aims for the next cycle were (a) maintenance of improvements in BCT (b) improve the uptake of the frontal test sheet to $100 \%$ if possible.

PDSA cycle 2: Given the success in PDSA cycle 1, the basic intervention was not changed, rather the same advice was reiterated and redistributed at team meetings, by email, and by individual discussion to address any questions or concerns. The same measures used in PDSA cycle 1 were collected for a month's worth of referrals (July 2015). We predicted we could maintain performance in the uptake of bedside cognitive testing.

The results were as follows. There were 15 referrals in total, with mean age 48.4 years, 10 (66.6\%) being male. Eleven (73.3\%) were suitable for testing although four $(36.4 \%)$ of these had insurmountable barriers to testing including severity of medical or 
psychiatric illness, patient refusal, lack of interpreter availability, and severe aphasia. Of the seven able and suitable referrals, 6 (85.7\%) had an ACE with no patients having a MoCA. Where applicable, the ACE cognitive subcategories were documented in all (100\%) cases. Six $(85.7 \%)$ had frontal testing, the frontal test sheet being used in five $(83.3 \%)$ of these cases.

At the end of this cycle and QI project, the ongoing aims were (a) maintenance of improvements in bedside cognitive testing (b) continued use of the ACE/MoCA with the frontal test sheet.

\section{Lessons and limitations}

Although these factors were not objectively measured, there were other competing forces at play which may have impacted on the comprehensiveness of bedside cognitive testing - for example, efficiency of service delivery with time being preferentially dedicated to other aspects of care (e.g. history, mental state examinations, collateral histories, interactions with family and medical teams, psycho-education, mental capacity testing, mental health act assessments, multi-disciplinary meetings). After discussion, this was the reason PDSA cycle 2 did not include further test sheets looking, for example, at parietal lobe function. At the time of writing, there were ongoing departmental discussions about the potential recruitment of a neuropsychiatry specialist nurse who could help facilitate more detailed bedside cognitive testing.

A further limitation is the low numbers of patients in the PDSA cycles. For this reason, the uptake of testing between PDSA cycle 1 and PDSA cycle 2 is unlikely to be statistically significant (the difference between $100 \%$ uptake in PDSA cycle 1 and $85.7 \%$ in PDSA cycle 2 was just one patient).

It was important to the clinical team that the results of this project were sustainable and that the new approach to bedside cognitive testing became a practical and manageable, mainstream way of working. By the end of this project, the test sheet introduced had become the standard way we conduct bedside cognitive testing in the department. Furthermore, this new way of working was able to withstand significant challenge and the variation in the number of referrals to our service. Due to new referral pathways (particularly with the stroke service), the number of referrals to the neuropsychiatry team tripled (number of patients per month) over the timeframe between baseline measurement and PDSA cycle 2. It was still however possible to improve bedside testing and largely maintain these improvements into PDSA cycle 2. The uptake was not however perfectly maintained (a $100 \%$ uptake of ACE or MoCA in PDSA cycle 1, for example, reduced to $85.7 \%$ in PDSA cycle 2). The comprehensiveness of frontal testing using the dedicated new test sheet nonetheless improved from cycle 1 to 2 , from $80 \%$ to $83.3 \%$.

There is no reason why the methods applied here cannot be applied in other centres, whether in the field of liaison psychiatry or on psychiatric wards where comprehensive bedside cognitive testing is seen as a priority (e.g. older adults inpatient wards where many patients with dementia are admitted). The methods here are therefore generalisable, as are the results if the methods are effectively implemented with consideration given to resources (staff time) and staff priorities.

\section{Conclusion}

In this project, careful thought and analysis of a team's existing practices with regards to bedside cognitive testing resulted in clear improvements in the uptake and comprehensiveness of bedside cognitive testing. This was done by the implementation of a formalised frontal lobe test sheet (with constituent tests described in 'Design' above) and clear guidance (having reviewed the literature) about the cognitive assessment tools that should be used.

The uptake of ACE (or MoCA) testing improved from $66 \%$ at baseline to $100 \%$ in PDSA cycle 1 . This was broadly maintained with an uptake of $85.7 \%$ in PDSA cycle 2. The uptake of frontal testing improved from $23.8 \%$ at baseline, to $100 \%$ in PDSA cycle 1 , falling slightly to $85.7 \%$ in PDSA cycle 2 . The comprehensiveness of frontal testing was greatly improved with $80 \%$ of patients in PDSA cycle 1 and $83.3 \%$ of patients in PDSA cycle 2 having detailed frontal testing using the test sheet implemented (compared with a baseline figure of $23.8 \%$ having very brief incomprehensive frontal testing).

It is the opinion of the author that these results are sustainable within the service and generalisable to other centres trying to improve the uptake and comprehensiveness of their bedside cognitive testing.

\section{References}

1 NICE. Nice Clinical Guideline 42. Dementia: supporting people with dementia and their carers in health and social care. Issued: November 2006 (last revised March 2015). Available at: http://www.nice.org.uk/guidance/cg42

2 Cullen B, O'Neill B, Evans JJ, Coen RF, Lawlor BA. A review of screening tests for cognitive impairment. J Neurol Neurosurg Psychiatry 2007;78:790-799.

3 The Montreal Cognitive Assessment (MoCA). Available at: http://www.mocatest.org

4 Folstein MF, Folstein SE, McHugh PR. "Mini-mental state". A practical method for grading the cognitive state of patients for the clinician". Journal of Psychiatric Research, 1975;12(3):189-198.

5 Feldman R, Newman J. Copyright at the bedside: should we stop the spread? Stanf Technol Law Rev 2013;16(3):623-655.

\section{Declaration of interests}

No conflict of interest declared.

\section{Acknowledgements}


Many thanks to Dr Sophie Epstein and Dr Mervi Pitkanen for their help and support.

\section{Ethical approval}

This project was an audit and quality improvement project. Ethical approval was therefore not required. 